\title{
A Model for Optimally Promoting Application Diffusion on Facebook
}

\author{
Guoying Zhang ${ }^{1}$, Chuck Johnston ${ }^{1} \&$ Chris Shao ${ }^{1}$ \\ ${ }^{1}$ Dillard College of Business Administration, Midwestern State University, TX, US \\ Correspondence: Guoying Zhang, Dillard College of Business Administration, Midwestern State University, \\ 3410 Taft Blvd., Wichita Falls, TX, 76310, USA. Tel: 1-940-397-3289. E-mail: grace.zhang@mwsu.edu
}

$\begin{array}{ll}\text { Received: February 28, } 2014 & \text { Accepted: April 14, } 2014 \quad \text { Online Published: July 28, } 2014 \\ \text { doi:10.5539/ijms.v6n4p35 } & \text { URL: http://dx.doi.org/10.5539/ijms.v6n4p35 }\end{array}$

\begin{abstract}
Facebook, the leading social networking site, has opened its platform to developers and allow them to publish applications. Subsequently, numerous Facebook applications of various types were designed and deployed. Despite a huge portion of applications without business context, there exist a substantially increasing number of applications tailored specifically for marketing and advertising. From a business perspective, a Facebook application possesses the advantages of low development costs and strong word-of-mouth effect, which provide an ideal alternative to traditional advertising formats. This paper utilizes the well-known Bass model for forecasting product diffusion, and proposes its adaptation to produce an optimal promotion budget allocation for Facebook applications. For a given application promotional budget to be used over a fixed timeframe, the model offers a unique solution for allocating the funds between direct promotion and indirect promotion (word-of-mouth) in order to achieve a maximum percentage of user installations from the target population of potential users. Numerical examples are provided to illustrate the optimal solution and suggestions are made for future research necessary to validate the model for possible use by practitioners.
\end{abstract}

Keywords: diffusion, Facebook application, Bass model, promotional strategy, budget allocation

\section{Introduction}

\subsection{Background}

Established in 2004, Facebook has become the premier social networking platform. A recent statistical report from Facebook claims that it has more than 800 million active users. In May, 2007 Facebook opened its platform to enable companies and developers to design applications that can be integrated with a Facebook website for user access. Virtually any organization can now design an application and make it available for download on the social network. These applications are commonly referred to as Facebook Apps. On average, users install Facebook applications more than 20 million times on a single day. Every month, more than 500 million people use a Facebook application. There are more than 7 million applications now integrated with Facebook (Facebook, 2012).

Although many Facebook applications feature music, online gaming, and other social activities, there are also applications tailored for marketing and advertising utilizing rich business contexts. From a business perspective, the exposure through social network applications can be an effective channel for conducting marketing campaigns, displaying advertising, and for paid sponsorships.

Due to the low entry barrier and low cost of developing Facebook applications, businesses are increasingly valuing the Facebook platform as an important channel in which to advertise and promote their brands. For example, to promote a new television series the A\&E Television Network built a "Parking Wars" Facebook application. With this application users parked virtual cars on the profile pages, or "streets," of friends and they also were able to issue tickets to those cars. After just three months, "Parking Wars" had over 198,000 unique users. Another example is from Sony. A popular application was used to promote a sweepstakes and attracted 1 million participates (King, 2008). Hagel and Brown (2008) concluded that Facebook applications provide "a new format of interactive communication and advertising." More recently, it is also noted that marketers are increasing their Facebook advertising budgets at a faster rate than they are for search ads. For the first half of 2012, one marketing firm clients' Facebook ad budgets are up 36\%, whereas paid search budgets are up 12\% (Darwell, 2012).

Facebook advertising gives companies the flexibility to manage targeted market segmentations. A Facebook 
member needs to join at least one social network, either geographical or educational. In addition, members provide personal profiles and preferences such as relationship status, hobbies, favorite celebrities, and daily activities, which allow businesses to use such information for targeted advertisements. Further, Facebook advertising has been shown to be less expensive than Google's AdWords, which can cost up to around $\$ 2$ per keyword per click. For example, Jepson (2009) reported that Truliant Federal Credit Union hit nine million views in seven months for about $\$ 5,000$ after advertising using a Facebook application.

The general life cycle of a Facebook application begins with the developer of the application publishing the application on Facebook. After that, a specific link for this application appears in the application directory. Users of Facebook can search for the application in the directory and install it simply by clicking the link and following the directed steps. After installation, each activity of the user associated with this application is reported as a mini-story feed to the user's friends. Friends of the user also get invitations from the user to install the application, or simply get curious about the application because of the mini-story feeds and install the application directly from the directory. This viral aspect of Facebook applications, which is highly valued by online advertisers, can easily diffuse advertising messages using the application through the network of friends. This sharing behavior through social network links is essentially the main driver of promoting Facebook application installations. How this viral aspect is modeled as the imitation effect of the application diffusion process is discussed later.

\subsection{The Diffusion Process}

The diffusion process is often defined as the "process by which an innovation is communicated through certain channels over time among members of a social system" (Rogers, 2003). With regard to the Facebook platform, it is proposed that applications be treated as innovation with the social system being the networks of friends in Facebook. In this exploratory effort, the number of application installations (downloads) is also established as a proxy for successful communication channels. Therefore, the diffusion process of Facebook applications having specific advertising messages is basically the process of getting more and more user installations over time.

The focus here is on considering the two different approaches leading to users' Facebook application installations. One approach is using the directory of Facebook applications, and the other is to acquire applications from news feeds or friend recommendations (see Appendix B for illustrations of each). The first approach is a direct approach and modeling it as the innovation effect of the diffusion process will be explained later. The second approach is an indirect approach and referred to hereinafter as the imitation effect. The diffusion process of Facebook applications is thus investigated taking into consideration both the innovation and imitation effects.

Although Facebook applications are downloaded millions of times each day, few formal studies exist attempting to establish a general diffusion process for these applications reaching users. Understanding the diffusion process is critical for businesses interested in investing in Facebook applications as a new social tool for advertising. With better knowledge of the diffusion process, applications developed primarily for viral advertising can be more efficiently deployed and measured for effectiveness over time.

\subsection{The Exploratory Model}

As previously noted in the discussion of the general life cycle of a Facebook application, the diffusion of a Facebook application to users is mainly realized in two ways: direct installation of the application through links in the application directory and indirect installation through friends' invitations. It is proposed that the utilization of these two channels by users can possibly be enhanced by active intervention from businesses in the form of promotional activities properly allocated between the channels, such as promoting applications in the application directory and giving rewards for inviting friends. In this exploratory effort, the Bass model (Bass, 1969) is adapted to model the diffusion process of Facebook applications. An optimal diffusion strategy for the promotional budget is also analytically developed in the context that businesses can maximize the user installation percentage of the target population for a specific Facebook advertising application, given the constraint of a promotional budget. It is believed that a balanced approach of optimally promoting both channels to improve the diffusion of Facebook advertising applications is desirable for businesses.

The paper proceeds as follows: Section 2 introduces related work in the field. The adapted Bass model is established in Section 3 with an analytical solution for optimally allocating resources to promote the diffusion of Facebook applications. Numerical examples are also developed to illustrate how to find the optimal allocations of promotional resources. Conclusions and suggestions for future research to validate the model are found in Section 4. 


\section{Selected Related Work}

The classic Bass diffusion model is adapted here to study the diffusion of Facebook applications. The way new products are adopted is described by this model as a process of interaction between users and potential users (Bass, 1969). Mahajan, Muller, and Bass (1990) conducted a comprehensive review of dozens of articles in the literature using the original Bass model. They categorized these into five research areas: basic diffusion, parameter estimation, flexible diffusion, refinements and extensions, and use of diffusion models. More specifically, for the use of diffusion models, they pointed out an area of normative study to derive optimal pricing, advertising, and timing strategies in monopoly and oligopoly markets. Our study intends to derive an optimal advertising budget allocation strategy, which fits into this research category among other related literature.

More recently, Bass (2004) discussed other important extensions of the original Bass model, examples of application, and examples of frontier research. The article provided additional insights into the properties of the model. We were also motivated by the research of Firth, Lawrence, and Clouse (2006). They applied the Bass Model to online communities for forecasting how many people will join the community and how long it will take to reach peak membership. It showed that the Bass Model can delineate two different types of influences (internal and external). Trusov, Bucklin, and Pauwels (2009) also quantitatively measured the effects of word-of-mouth marketing on membership growth at an Internet social networking site and compared it with traditional marketing effects. Results indicated that word-of-mouth referrals had longer carryover effects than traditional marketing actions. The Bass diffusion model was one of several models used in this research.

The Bass model has also been extensively used to forecast new product and technology diffusion. The works cited in this section are not intended to be a comprehensive review of research related to the development of the Bass model. This vast body of literature is readily available to the interested reader. As stated above, this paper is focused on the adaptation of the model to the diffusion of Facebook applications. Therefore, the presentation of the Bass model covers its essential constructs and assumes a limited familiarity with its development that can easily be supplemented from other sources with additional background material as desired and required by the reader.

Many studies have been conducted related to Facebook and to the Internet as marketing tools for organizations and businesses. Some of these are briefly listed to illustrate their general nature compared to this effort to deal more specifically with the use of Facebook applications for advertising purposes and how they could possibly be more efficiently and effectively promoted.

Mazzocchi (2008) discussed the successful use of Facebook by San Diego State University to attract promising students. Fou (2008) compared the online advertising benefits associated with cost-per-thousand-impressions advertising, cost-per-click advertising, and cost-per-action advertising. Using social media as a business tool for improving marketing efforts was suggested by Jacobs (2009). Jepson (2009) reported a successful use of Facebook to implement an advertising campaign. Smith (2009) focused on the establishment of online social media to promote business. Shafer (2010) introduced the advantages for sporting goods dealers to leverage the power of the Internet in selling. Arnold (2009) argued that social networking sites are significant marketing vehicles and cost effective tools for advertising. York (2010) explained how a coffee chain incorporated a social media strategy in marketing their products for increased profits and to create more traffic in their stores.

Much more closely related to this research, Truong (2008) studied a self-developed Facebook application. With a 648-user application, data were collected in terms of application installation, user information, and recommendations to friends. The adoption rates of this specific application show that most users find it in the application directory, and not by word-of-mouth referrals. The author admitted that further facilitating this kind of growth would require applications showing in users' "news feeds" when they are installed. Chu (2011) investigated the potential relationship between Facebook group participation and viral advertising responses. His findings suggest more positive attitudes toward advertising and social media among college-age Facebook group members than among non-group members.

There are also numerous research studies in the marketing literature on advertising using viral marketing. One of the studies concerns the effectiveness of viral marketing. Leskovec, Adamic, and Huberman (2007) explored the effectiveness of peer recommendations. The study obtained a counterintuitive result that as peer recommendations increase; they actually lower the probability of a purchase. The maximum purchase probability is reached when there are exactly two recommendations. Online commenting, peer recommendations, and other digital formats of viral marketing make the evaluation of this advertising approach feasible. Therefore, it is also reasonable to assume that viral marketing, when optimally allocated, could help the diffusion of Facebook 
applications.

\section{An Optimal Diffusion Model}

As mentioned in the Introduction, due to the low cost of creating and hosting applications on the Facebook platform, in addition to the attractiveness of the large potential audience, businesses are seeking advertising solutions utilizing Facebook applications to raise brand awareness and engage audiences. There are many so-called organic-grown applications on Facebook. This means that those applications follow a natural diffusion pattern to gain user installations. However, this preliminary effort is more interested in how to potentially intervene and improve the diffusion process of these applications from a business advertising perspective rather than to attempt to explain every facet of what Facebook may be doing at a particular moment in time that could affect both the direct (innovation effect) and indirect (imitation effect) diffusion processes. Like any technology based product, the Facebook platform is constantly changing and evolving. This "optimal diffusion model" would have to be validated using empirical data derived from its application in a particular set of circumstances. This "optimal diffusion model" is therefore broadly conceived and intended to be broadly applied and tested to determine its usefulness as a specific or general guideline/tool. To that end, it is first required that we establish how this diffusion process, as represented by the Bass (1969) model, can be related to the propagation of Facebook applications over a time-based promotional campaign.

\subsection{Using the Bass Model}

The diffusion model first developed by Frank Bass describes a process of product adoption resulting from interactions between current users and potential users (Bass, 1969). Bass validated his model using data for various product introductions that took place over long periods of time. According to the Bass model, there are two factors enabling product adoption, namely, innovation effect and imitation effect. Innovation effect refers to direct adoption of the product, e.g. independent of the existing adoptions. On the other hand, imitation effect means adopting the product based on imitating other users, e.g. dependent on the existing users.

In this study, a Facebook application is viewed as a new product and the installation of the application corresponds to product adoption. In fact, only a small number of Facebook users will be able to identify the application organically without the developer's promotion. In order to facilitate and improve the diffusion of Facebook applications, there are two possible approaches; one is to promote a direct installation from the App Center or the App Directory (the innovation effect of the Bass model).

\subsection{The Innovation Effect}

Promoting applications on Facebook is similar to promoting a webpage or a product. Advertisers can bid on the sponsor lists in specified slots on the Facebook platform, or other online advertising vehicles, to attract direct clicks by users. The cost of such an approach is in general either in the form of traditional CPC (cost per click) or CPM (cost per thousand impressions). More recently, in December 2012, Facebook specifically coined a "Promote Your App" page for application developers. This function allows developers to promote application installations mainly through mobile app install ads, which appear in mobile news feeds to take people directly to the App Store to install the app, and sponsored results, which provides developers with the ability to buy ads in search results (Cohen, 2012).

\subsection{The Imitation Effect}

Another way to promote installations of Facebook applications is to utilize the word-of-mouth features of this social network platform. Here in this exploratory research, it is therefore reasonable to equate this indirect approach to the imitation effect of the Bass model where new adopters are dependent on previous adopters. With Facebook applications, recommending an application to friends is a significant expectation of user activities. A simple example can be used to describe such recommendations. Suppose user A finds a BMW drive application via the application directory and decides to install it. During the installation, the application will prompt user A with a choice to invite friends for a virtual ride together, or to have a race. User A can decide which friend(s) to invite, if any, to join the activity. Of course the friend has to first install the application before being able to participate. This installation activity then can possibly also generates a mini-story feed, which is pushed to the Facebook home page of each friend of user A. Friends who were chosen by user A to join the activity could further receive additional invitation notifications. Suppose user B, who receives an invitation from user A, decides to install the application. A similar recommendation procedure could then be repeated and propagated to the network of friends of user B. Eventually, all of the related users' activities with the application could be recorded and fed to the networks of their friends.

The imitation effect is the most difficult with which to deal due to the constant changes that may be incorporated 
into how Facebook chooses to provide "suggestions" to other users. When Facebook does this, it serves to emphasize that in this day of constant searching for ways to improve the effectiveness of marketing and advertising efforts, change in the status quo is inevitable. A more recent development in this area is what has been termed "social filtering" wherein Facebook can intervene in the newsfeed process to influence what is seen by friends of users. Social filtering and any other major changes by Facebook of course have the potential to render any initially developed diffusion model obsolete by causing the imitation effect to be overestimated, or perhaps underestimated. In addition to "social filtering," the interpersonal flow of communication may cause an overestimated/underestimated imitation effect as well. As suggested by Lazarsfeld, Berelson, \& Gaudet (1944) in the multi-step flow of communication theory, communication process takes into account the fact that information and influence are often two-way in which "opinion leaders" both influence and are influenced by opinion receivers. It is because of this reciprocity of information overestimation or underestimation occurs. However, this is a risk for any modeling effort and would have to be monitored going forward in the validation and use of the model. Every major change by Facebook would warrant re-evaluation of the model using empirical data, especially the validity of the imitation effect as currently represented. The initial model is just a beginning. It is intended to be dynamic in nature, not static.

\subsection{Enhancing the Effects}

To take advantage of the opportunity to enhance the diffusion of Facebook applications in this manner, organizations promoting applications for advertising purposes can encourage developers to create more viral features in them with strong social networking characteristics. For example, TravelBrain used to provide sweepstakes opportunities for users who fulfilled five activities in order to enhance the diffusion of the application through the indirect viral channel. These activities included recommending a place to travel, sharing travel tips, writing a review about a place and inviting friends to rate the review. Organizations can also provide incentives to users for making recommendations to friends and engaging in more activities to generate feeds that will be sent to friends.

Given these two different approaches for promoting Facebook applications for advertising purposes, this exploratory effort proposes a possible model to answer the question; "For a given amount of advertising resources (such as a budget), what is the optimal allocation of resources between direct promotion (innovation) and indirect promotion (imitation), such that the Facebook application will be downloaded by a maximum percentage of the target population within a given time period?"

\subsection{Promoting Facebook Applications}

Promotional campaigns are often constrained by resources, most often by a monetary budget. Assuming an organization is interested in developing a Facebook application to advertise one of its services or products, the traditional approach is to choose a direct channel and post the advertisement. Hence, the entire advertising budget is used for this, or some combination of direct channels. There also exist traditional advertising programs to promote products using word-of-mouth, or referral, incentives. However, the incentives are usually realized only with final purchase of the product or service instead of just for being exposed to the advertisement.

With the increasing prominence of Facebook, online advertising is also important to organizations. GM's advertising expenditure on Facebook was approximately $\$ 10$ million in 2011. Compared to their total U.S. ad spending of $\$ 1.8$ billion (Kantar Media, 2012), it is a relatively small amount, but still significant. GM also started to re-evaluate its Facebook advertising earlier in 2012 in order to increase the effectiveness of the ads. Additionally, within the auto industry, Subaru of America committed to more than $\$ 5$ million in ad spending in 2012 on Facebook and the spending will increase if the return is effective (Terlep, Vranica, \& Raice, 2012). Facebook's total 2011 revenue was $\$ 3.7$ billion, most of which was advertising sales.

Therefore, it is unique for Facebook applications to serve as advertising media where the viral word-of-mouth effect of advertising (instead of purchasing) can be clearly tracked and managed. With the availability of more information provided by the online environment in which Facebook applications are dispersed, come opportunities to perhaps optimize the allocation of our constrained promotional resources between the direct channel - innovation effect, and the indirect channel -imitation effect.

\subsection{Model Development}

Bass's 1969 model has been widely adapted for many purposes including estimating the diffusion pattern of innovations and new products. Some preliminary justification has already been provided for using this model to capture the diffusion process of Facebook applications. To further that process, it is assumed that the $S$-curve is appropriate to model the installations of a Facebook application over time. When introduced, the applications are 
new products and the $S$-curve can be used to represent the adoption (installation) of the applications. A brief explanation of the Bass model is provided prior to illustrating how it is adapted to the diffusion process for Facebook applications in order to optimize the allocation of a promotional budget.

\subsubsection{The Foundational Basis}

The Bass model has at its core two previously mentioned effects: the innovation effect - the direct adoption rate by users, and the imitation effect - the adoption rate of additional users triggered by interaction with existing adopters. Bass begins with the basic idea that the probability of an initial purchase at time $T$, given no previous purchase is:

$$
P(T)=p+(q / m) Y(T),
$$

where $p$ and $q / m$ are constants and $Y(T)$ is the number of previous buyers. Further, since at $T=0, Y(T)=0, p$ is the probability of an initial purchase with its magnitude then reflecting the importance of innovator purchasing. Obviously, the selection of an appropriate time scale measurement is critical to the validity of the model in any applied sense. This is discussed later in the paper. Therefore, $m$ simply represents the number of initial purchases over the time period of interest.

An additional assumption of the Bass model (that fits well with the current consideration of Facebook applications as a product) is restricting its interest to only initial sales; excluding "replacement" sales of the product such as can be the case with more durable consumer goods. Therefore, Bass' initial statement of the likelihood of a purchase at time $\mathrm{T}$ is given as;

$$
[f(T)] /[1-F(T)]=P(T)=p+q / m Y(T)=p+q F(T),
$$

and at any given point in the time interval of interest, $T$, the total number of purchases of the product is then represented by:

$$
F(T)=\int_{0}^{T} f(t) d t,
$$

Bass went on to summarize additional assumptions associated with his model development in terms of "behavioral rationale." Both "innovators" and "imitators" will initially purchase the product. However, an innovator will buy the product independently and directly, while an imitator is influenced by the number of previous buyers. The importance of innovators in this process will also decrease with time.

These two different types of purchasing are represented in the Bass Model by an innovation effect, $p$, and an imitation effect, $q$. Thus, the probability of purchasing at time T, $f(\mathrm{~T})$, is expressed by Bass as:

$$
f(T)=(p+q F(T))(1-F(T))=p+(q-p) F(T)-q[F(T)]^{2},
$$

where $F(\mathrm{~T})$ is the cumulative density function, or the total number of purchases at time $\mathrm{T}$. In order to solve for $F(\mathrm{~T}), f(T)$ in the above equation is replaced with $\frac{d F(T)}{d T}$ :

$$
\frac{d F(T)}{d T}=p+(q-p) F(T)-q[F(T)]^{2}
$$

Solving the above differential equation with a boundary condition of $F(0)=0$, the total number of purchases from time 0 to $T$ can be expressed as (See Appendix A for the solution):

$$
F=\frac{1-e^{-(p+q) T}}{1+\frac{q}{p} e^{-(p+q) T}} .
$$

\subsubsection{The Maximization Goal}

Placed within the context of Facebook applications used for advertising purposes, a worthwhile goal for an organizational advertiser would be to maximize the total number of installations (downloads) of its Facebook application within a certain time period, $T$, and within its fixed promotional budget. Using the above formula and normalizing the promotional budget to 1 results in the total number of installations at time $T$, i.e., the total adoption of a new product at time $T$, being: 


$$
F=\frac{1-e^{-(p(k)+q(1-k)) T}}{1+\frac{q(1-k)}{p(k)} e^{-(p(k)+q(1-k)) T}},
$$

where $p(k)$ denotes the innovation effect being a function of promotional spending $k$ out of the total budget 1 , $0 \leq k \leq 1$; and $q(1-k)$ denotes the imitation effect being a function of the remaining budget $(1-k)$. Both the innovation and imitation effects are now functions of promotional spending. By interpreting the model in this manner, spending money on directly promoting the application can immediately increase the innovation effect while also increasing the viral word-of-mouth/imitation effect by providing monetary incentives with the remainder of the promotional budget. The goal is to find a balance point to allocate the promotional resources/budget effectively.

\subsubsection{Effect Characteristics Assumptions}

It is also assumed that the innovation (direct promotion) and imitation (indirect/word-of-mouth) promotional spending functions share common characteristics: the greater the promotional spending, the larger the corresponding effect (innovation or imitation). However, at the same time, the marginal increase of the effect is shrinking with additional spending. In other words, the relationship between promotional spending and the corresponding effect would follow the shape of a non-decreasing and concave production function. A production function usually depicts the relationship between quantities of input and the output that can be produced. A common production function with one input factor is illustrated in the following figure:

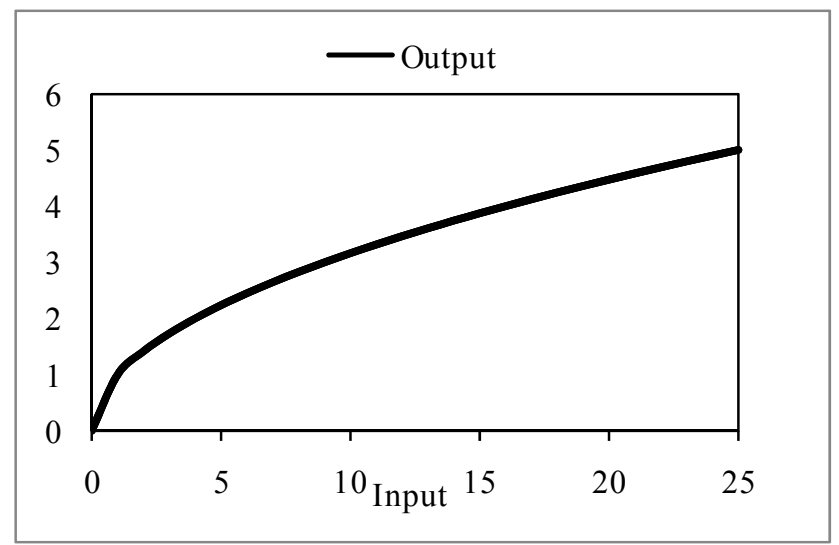

Figure 1. A production function example modeling the assumed relationship between promotional spending and resulting adoption rates (innovation and imitation effects)

\subsubsection{Maximization within Timeframe and Budget Constraints}

Therefore, based on the above assumptions, the management decision problem then becomes to maximize the total number of installations of a new Facebook application within a time period $T$, given a fixed budget amount. More specifically, the maximization problem can be modeled as:

$$
\max _{k} F(T)=\frac{1-e^{-(p(k)+q(1-k)) T}}{1+\frac{q(1-k)}{p(k)} e^{-(p(k)+q(1-k)) T}}
$$

$$
\text { s.t. } 0 \leq k \leq 1
$$

In this maximization problem, the decision variable is $k$, representing the portion of promotional spending allocated to direct methods, the innovation effect, $p(k)$. Accordingly, the remaining budget, 1- $k$, is spent on promoting word-of-mouth type efforts, the imitation effect $q(1-k)$. Both $p(k)$ and $q(1-k)$ are assumed to have a functional form similar to the production function illustrated in Figure 1. Hence, the above maximization problem is solved to obtain an optimal allocation of the promotional budget. 
Proposition 1. For a given time period T, the optimal allocation of the promotional budget between activities enhancing innovation and imitation effects should satisfy:

$$
\frac{p^{\prime}\left(k^{*}\right)}{q^{\prime}\left(1-k^{*}\right)}=p\left(k^{*}\right) \frac{\left(\left(p\left(k^{*}\right)+q\left(1-k^{*}\right)\right) T-1\right)+e^{-\left(p\left(k^{*}\right)+q\left(1-k^{*}\right)\right) T}}{\left(p\left(k^{*}\right)\left(p\left(k^{*}\right)+q\left(1-k^{*}\right)\right) T+q\left(1-k^{*}\right)\right)-q\left(1-k^{*}\right) e^{-\left(p\left(k^{*}\right)+q\left(1-k^{*}\right)\right)^{T}}} .
$$

Proof: as stated above, we need to find an optimal portion $k$ of the total budget to maximize total installations, $F$ :

$$
\max _{k} F=\frac{1-e^{-(p(k)+q(1-k)) T}}{1+\frac{q(1-k)}{p(k)} e^{-(p(k)+q(1-k)) T}} ; \text { s.t. } 0 \leq k \leq 1 .
$$

Taking the first order condition with regard to $k$ yields:

$$
\frac{\partial F}{\partial k}=\frac{\partial F}{\partial p} p^{\prime}(k)-\frac{\partial F}{\partial q} q^{\prime}(1-k)
$$

letting the first order condition $\frac{\partial F}{\partial k}$ equal zero:

$$
\frac{p^{\prime}\left(k^{*}\right)}{q^{\prime}\left(1-k^{*}\right)}=\frac{\partial F}{\partial q} / \frac{\partial F}{\partial p}=p\left(k^{*}\right) \frac{\left(\left(p\left(k^{*}\right)+q\left(1-k^{*}\right)\right) T-1\right)+e^{-\left(p\left(k^{*}\right)+q\left(1-k^{*}\right)\right) T^{T}}}{\left(p\left(k^{*}\right)\left(p\left(k^{*}\right)+q\left(1-k^{*}\right)\right) T+q\left(1-k^{*}\right)\right)-q\left(1-k^{*}\right) e^{-\left(p\left(k^{*}\right)+q\left(1-k^{*}\right)\right) T}}
$$

Therefore, Proposition 1 shows that there exists a unique solution for optimally allocating the promotional budget.

3.6.5 Applying the Results

A simple numerical example can help to visualize the result. Let $p(x)=q(x)=\sqrt{x}$, and thus satisfying the requirement of following the shape of a non-decreasing and concave production function. Figure 2 presents the curve representing the partial derivative of the total number of installations with regard to $k, \partial F / \partial k$. The optimal $k^{*}$ is obtained when this curve intersects the horizontal axis. In Figure 2, each plot indicates a given time period $T$ and the corresponding optimal allocation of the total budget, $k^{*}$.

From Figure 2, the model suggests that as the fixed time period $T$ increases, consideration should be given to shifting the promotional focus from enhancing the direct innovation effect to encouraging the word-of-mouth indirect effect. In other words, for a Facebook application promotion, the longer the planned promotional period for diffusion the greater the proportion of the promotional budget that would be spent on enhancing the word-of-mouth effect. An example of insights derived here can be stated as: applications advertising for fast consumption products, such as fashion products, electronics, or food products, are more effective with greater budgeting for the innovation effect; while applications advertising for products allowing longer time periods for promotion and diffusion, such as travel and leisure, banking offerings, or vehicles, are more effective with a greater proportion of budgeting for the imitation effect. 


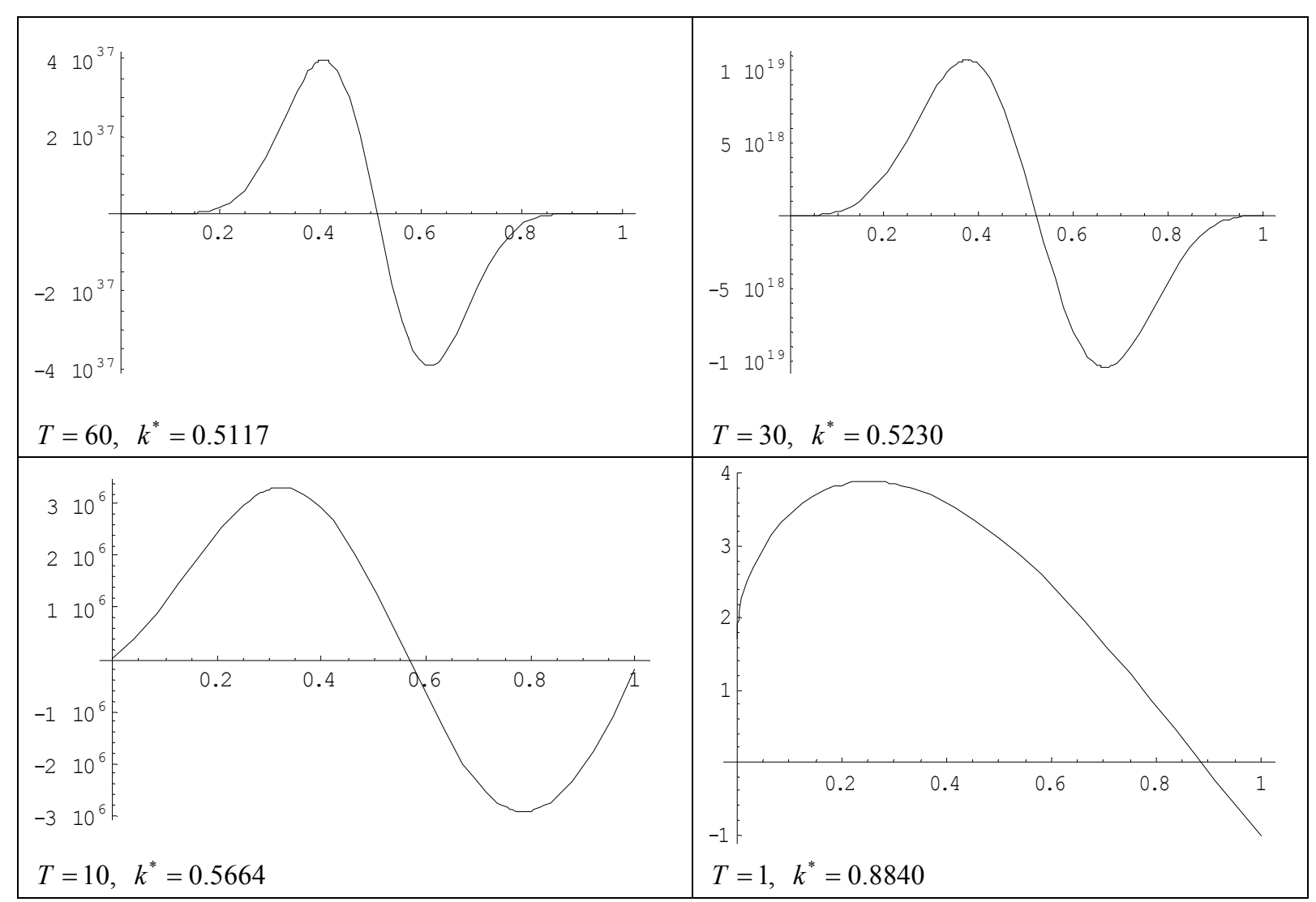

Figure 2. Visualization of equation (1) as a function of $k$, the portion of promotional spending allocated to direct methods (the innovation effect)

By fitting a power trendline to the results of Figure 2 (Figure 3), it is possible to illustrate this trend in the budgeted direct promotion allocation, $k^{*}$, for increasing time periods, $T$, and to facilitate calculations for discussion and for possible use by practitioners. From this trendline, it can be observed that the portion of the promotional budget invested in the direct innovation effect will probably remain above $50 \%$, depending on how the time period, $T$, is eventually defined, and may be especially important for short promotional efforts. At the same time, the trendline indicates that the indirect/word-of-mouth imitation effect is potentially equally critical for the application diffusion, as the promotional time period lengthens. Overall, as long as the relationship between promotional spending and the corresponding effect follows the shape of a non-decreasing and concave production function, advertisers desiring to promote their Facebook applications can possibly use these results to derive optimal budget allocations and thereby enhance the diffusion of their applications. 


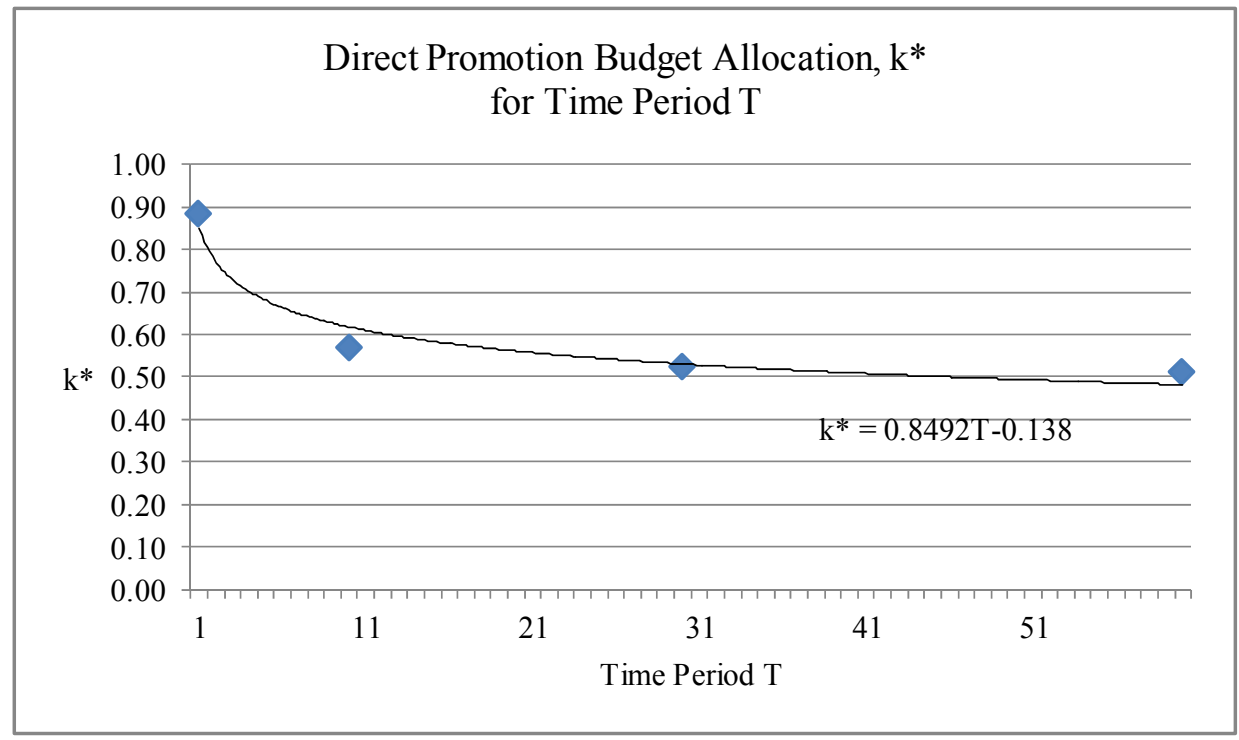

Figure 3. Direct promotion budget allocation for time period T

\section{Discussion and Future Research Directions}

Marketers continue to increase the portion of their advertising budgets allocated for Facebook. At the same time, they need a better understanding of how to most effectively utilize the Facebook advertising budget. Two aspects of this model become increasingly significant for use by practitioners; how is the time period, $T$, to be defined (i.e. hours, days, weeks, months), and for what size promotional budgets is it most appropriate. The following table was developed using the trendline formula to facilitate the discussion of these issues.

Table 1. Sample optimal budget allocation

\begin{tabular}{|c|c|c|c|c|c|c|c|c|}
\hline \multicolumn{9}{|c|}{ Budget } \\
\hline & & & $\$ 5,000$ & & $\$ 20,000$ & & $\$ 50,000$ & \\
\hline \multicolumn{9}{|l|}{ Time } \\
\hline Period, $T$ & Direct, $\mathrm{k}^{*}$ & Indirect, 1-k* & Direct & Indirect & Direct & Indirect & Direct & Indirect \\
\hline 1 & 0.8492 & 0.1508 & $\$ 4,246$ & $\$ 754$ & $\$ 16,984$ & $\$ 3,016$ & $\$ 42,460$ & $\$ 7,540$ \\
\hline 5 & 0.6801 & 0.3199 & $\$ 3,400$ & $\$ 1,600$ & $\$ 13,601$ & $\$ 6,399$ & $\$ 34,003$ & $\$ 15,997$ \\
\hline 10 & 0.6180 & 0.3820 & $\$ 3,090$ & $\$ 1,910$ & $\$ 12,361$ & $\$ 7,639$ & $\$ 30,902$ & $\$ 19,098$ \\
\hline 20 & 0.5617 & 0.4383 & $\$ 2,808$ & $\$ 2,192$ & $\$ 11,233$ & $\$ 8,767$ & $\$ 28,083$ & $\$ 21,917$ \\
\hline 30 & 0.5311 & 0.4689 & $\$ 2,655$ & $\$ 2,345$ & $\$ 10,622$ & $\$ 9,378$ & $\$ 26,554$ & $\$ 23,446$ \\
\hline 60 & 0.4826 & 0.5174 & $\$ 2,413$ & $\$ 2,587$ & $\$ 9,653$ & $\$ 10,347$ & $\$ 24,132$ & $\$ 25,868$ \\
\hline
\end{tabular}

Validating the model for use will require either access to past Facebook applications promotional budgets or using it for the allocation of future promotional budgets. Of these two alternatives, use in future promotional efforts seems the most practical unless applicable data might be available within organizations having previously developed numerous Facebook applications (An extensive search of the literature for any information on budgeting for Facebook application promotion produced no usable data with which to evaluate the model and it was deemed to be beyond the scope of this preliminary, exploratory effort to attempt to work with business organizations to develop the necessary data.).

The above table shows allocations for speculative small, medium, and large promotional budgets to be applied to efforts conducted over various time periods, $T$. In the rapidly moving online environment, it is suggested that $T$ be initially considered as days, weeks, or months. The extremes of either hours or years would not seem to be appropriate. Similar applications with comparable overall budgets (small, medium, or large), but with varying promotional timeframes, could have their allocations set according to the model and the resulting number of adoptions (downloads) compared for model fit. This is analogous to the approach used by Bass to validate his model which was intended to be used for sales forecasting. Regression estimates of the model parameters were 
developed using durable goods time series data. However, it must be noted that Bass was dealing with "infrequently purchased products" and time, $T$, was measured in terms of years ranging from 14 years for electric refrigerators and home freezers to 11 years for clothes dryers and steam irons. It is possible that the model when applied to promoting Facebook applications works best for large budget and relatively long timeframe ( $T=$ weeks, or months) efforts, or perhaps small budget and relatively short timeframes ( $T=$ days).

It is of course recognized that there are many other factors that could influence the validity of this simple model. Things such as the nature of the Facebook application itself, the targeted audience, the specific direct and indirect promotional vehicles chosen, and the changing policies and procedures of Facebook with respect to outside developed applications could all come into play. However, this research presents a potentially useful theoretical framework to model Facebook application diffusion over a targeted network. More importantly, it suggests a unique strategy for advertisers in the context of how to balance promotional resources to maximize the propagation of application installations. The classical Bass diffusion model may be sufficient to describe the diffusion of Facebook applications. With a given amount of promotional resources and a given time duration, an advertiser can postulate a unique balancing point to allocate resources to enhance both the innovation (direct) and imitation (indirect) effects of the diffusion process in order to maximize the total number of user installations in a potential market.

Finally, there are numerous other future research directions possible. First, only maximizing the total number of application installations was considered as the goal of promotional budget allocation. In reality, there are alternative measurements to consider. One of them is the number of monthly active users of an application. Facebook keeps track of not only the total installations of an application, but also the number of users who use the application every day. This measure should be able to capture the repeated exposure to a specific advertising message instilled in an application and may be as important as the total number of installations. Second, when using the Bass diffusion model, it is assumed that both innovation (direct) and imitation (indirect) effects are simply functions of the promotional budget. In fact, it is possible that these effects also vary over time. A dynamic programming model should be able to capture the effects of the time dimension and provide optimal solutions along a time path. Furthermore, a homogenous network structure of Facebook friends is implied when modeling the diffusion process. However, some recent studies point out that social networks are often heterogeneous. In other words, the innovation and imitation effects might be node specific as previously suggested. Lastly, it is also important to investigate other social media platforms with respect to advertising management. Incorporating some, or all, of these factors into specific variations of the proposed model should enable a more in-depth analysis of the diffusion pattern of interest.

\section{References}

Arnold, S. E. (2009). Social Media. Smart Business St. Louis, 2(11), 7.

Bass, F. (1969). A new product growth model for consumer durables. Management Science, 15(5), 215-227. http://dx.doi.org/10.1287/mnsc.15.5.215

Bass, F. M. (2004). Comments on "a new product growth for model consumer durables the bass model". Management Science, 50(12_supplement), 1833-1840. http://dx.doi.org/10.1287/mnsc.1040.0264

Chu, S. C. (2011). Viral Advertising In Social Media: Participation In Facebook Groups And Responses among College-Aged Users. Journal of Interactive Advertising, 12(1), 30-43. http://dx.doi.org/10.1080/15252019.2011.10722189

Cohen, D. (2012). Facebook Launches Promote Your App Page For Developers, Highlighting Advertising Options. All Facebook. Retrieved March 29, 2014, from http://allfacebook.com/promote-your-app_b106724

Darwell, B. (2012). Facebook ad budgets increasing faster than search budgets, Kenshoo study finds. Inside Facebook. Retrieved March 29, 2014, from http://www.insidefacebook.com/2012/08/24/facebook-ad-budgets-increasing-faster-than-search-budgets-ke nshoo-study-finds/

Facebook. (2012). Statistics. Retrieved January 7, 2012, from http://www.Facebook.com/press/info.php?statistics

Firth, D. R., Lawrence, C., Clouse, S. F., \& Koohang, A. (2006). Predicting Internet-based Online Community Size and Time to Peak Membership Using the Bass Model of New Product Growth. Interdisciplinary Journal of Information, Knowledge \& Management, 1. Retrieved from http://ijikm.org/Volume1/IJIKMv1p001-012_Firth01.pdf

Fou, A. (2008). Social Media Ads Are DOA. Media Week, 18(36), 14-15. 
Hagel, J., \& Brown, J. S. (2008). Life on the Edge: Learning from Facebook. Bloomburg Businessweek. Retrieved March 29, 2014, from http://www.businessweek.com/innovate/content/apr2008/id2008042_809134.htm

Jacobs, D. G. (2009). Surviving the Social Explosion. Landscape Management, 48(12), 10-13.

Jepson, K. (2009). As Marketing Budgets Get Slashed, 1 CU Finds Facebook Ads Prove ROI. Credit Union Journal, 13(12), 16.

Kantar Media. (2012). Kantar Media Reports U.S. Advertising Expenditures Increased 0.8 Percent In 2011, Kantar Media. $\quad$ Retrieved March 11, 2013, from $\mathrm{http}$ //kantarmediana.com/intelligence/press/us-advertising-expenditures-increased-08-percent-2011

King, R. (2008). Building a Brand with Widgets. Bloomburg Businessweek. Retrieved March 29, 2014, from http://www.businessweek.com/technology/content/feb2008/tc20080303_000743.htm

Lazarsfeld, P. F., Berelson, B., \& Gaudet, H. G. (1944). The People's Choice: How the Voter Makes up His Mind in a Presidential Campaign. Columbia University Press.

Leskovec, J., Adamic, L. A., \& Huberman, B. A. (2007). The Dynamics of Viral Marketing. ACM Transactions on the Web, l(1). http://dx.doi.org/10.1145/1232722.1232727

Mahajan, V., Muller, E., \& Bass, F. M. (1990). New product diffusion models in marketing: A review and directions for research. The Journal of Marketing, 1-26. http://dx.doi.org/10.2307/1252170

Mazzocchi, S. (2008). Schools turn to Facebook ads to recruit students. Advertising Age, 79(32), 20.

Rogers, E. M. (2003). Diffusion of Innovations (5th ed.). New York, NY: Free Press.

Shafer, T. (2010). Digital diversions. Dealernews, 46(7), 40.

Smith, H. (2009). Building Social Media Platforms for Growing Brands. Global Cosmetic Industry, 177(7), 26-28.

Terlep, S., Vranica, S., \& Raice, S. (2012). GM Says Facebook Ads Don't Pay Off. The Wall Street Journal. Retrieved March 29, from http://online.wsj.com/article/SB10001424052702304192704577406394017764460.html

Truong, H. (2008). Network Structure, Diffusion and Growth over Time in a Facebook Application. Working Paper. Retrieved from http://www-personal. umich. edu/ ladamic/courses/si508f07/projects/Facebook. pdf

Trusov, M., Bucklin, R. E., \& Pauwels, K. (2009). Effects of word-of-mouth versus traditional marketing: findings from an internet social networking site. Journal of marketing, 73(5), 90-102. http://dx.doi.org/10.1509/jmkg.73.5.90

York, E. B. (2010). Starbucks Gets Its Business Brewing Again With Social Media. Advertising Age, 81(8), 34. 


\section{Appendix A}

\section{Details of solving $\boldsymbol{F}(\mathrm{T})$}

Solving for $F(\mathrm{~T})$ from:

$$
\frac{d F(T)}{d T}=p+(q-p) F(T)-q[F(T)]^{2}
$$

Substituting $F(T) \equiv y$, the above equation can be rewritten as:

$$
\frac{d y}{d T}=(q y+p)(1-y),
$$

which is equivalent to:

$$
\frac{d y}{(q y+p)(1-y)}=d T \text {. }
$$

Integrating both sides yields:

$$
\begin{aligned}
& \int_{y} \frac{d y}{(q y+p)(1-y)}=\int_{T} d T \\
& \Leftrightarrow \frac{1}{1+p / q} \int_{y}\left(\frac{1}{(q y+p)}+\frac{1 / q}{1-y}\right) d y=\int_{T} d T \\
& \Leftrightarrow \frac{1}{p+q}(\ln (q y+p)-\ln (1-y))=T+C .
\end{aligned}
$$

Since at time 0 there are no installations, i.e., $F(0) \equiv y_{0}=0$, it is possible to solve for the constant term, $C=\frac{\ln p}{p+q}$ and the above derivation can be continued as:

$$
\begin{aligned}
& \frac{1}{p+q}(\ln (q y+p)-\ln (1-y))=T+C \\
& \Leftrightarrow \frac{1}{p+q}(\ln (q y+p)-\ln (1-y))=T+\frac{\ln p}{p+q} \\
& \Leftrightarrow \ln \frac{q y+p}{1-y}=(p+q) T+\ln p \\
& \Leftrightarrow \frac{q y+p}{1-y}=p e^{(p+q) T} \\
& \Leftrightarrow y=\frac{p e^{(p+q) T}-p}{p e^{(p+q) T}+q} \\
& \Leftrightarrow y=\frac{1-e^{-(p+q) T}}{1+\frac{q}{p} e^{-(p+q) T}} .
\end{aligned}
$$

Therefore, the equation is solved for $y$ and is substituted for $F(\mathrm{~T})$ in the paper. 


\section{Appendix B}

\section{Screenshots of Facebook Application Directory}

General Application Directory (available without signing in)

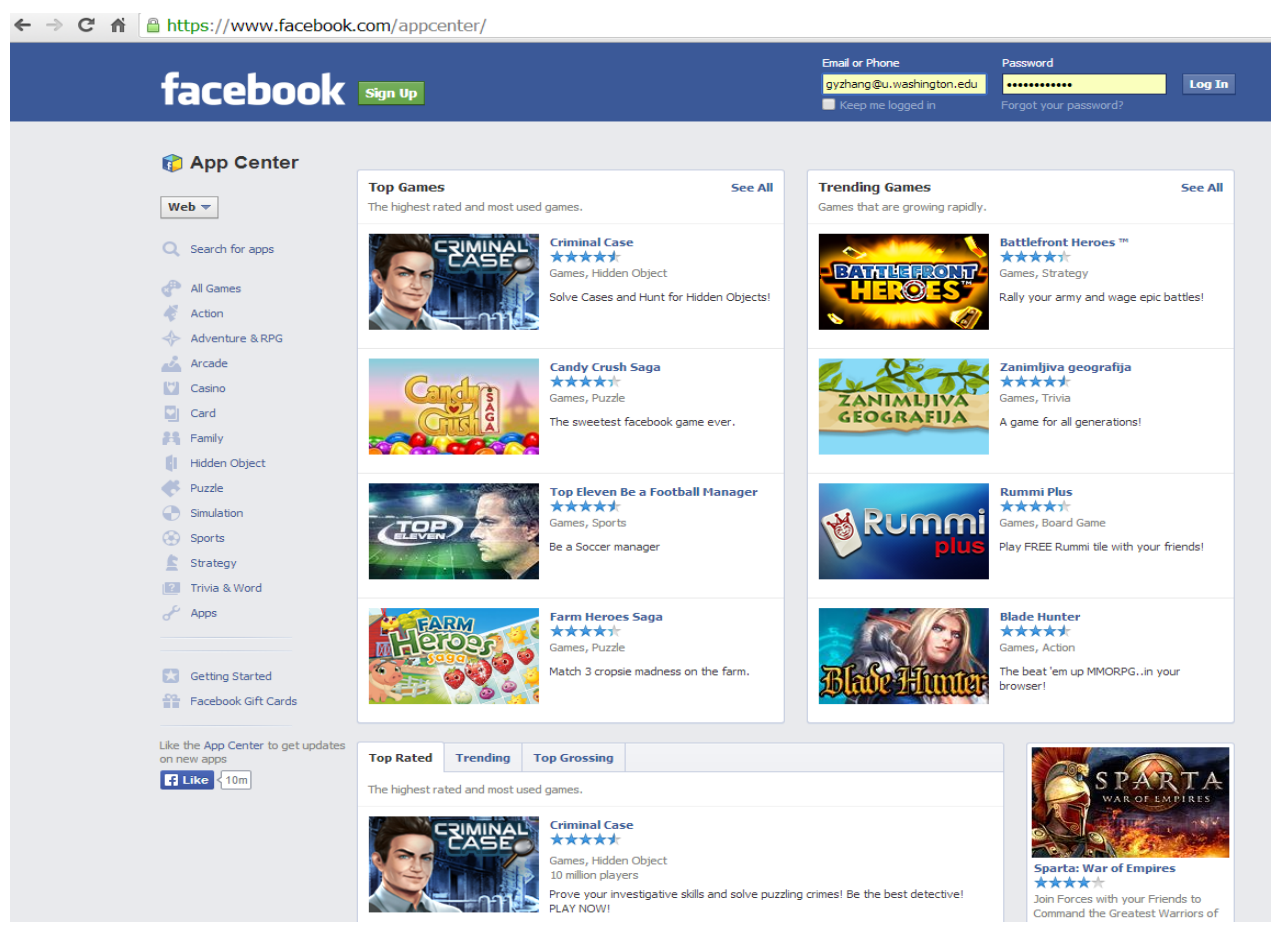

Directory including Friends' Applications (available when signed in)

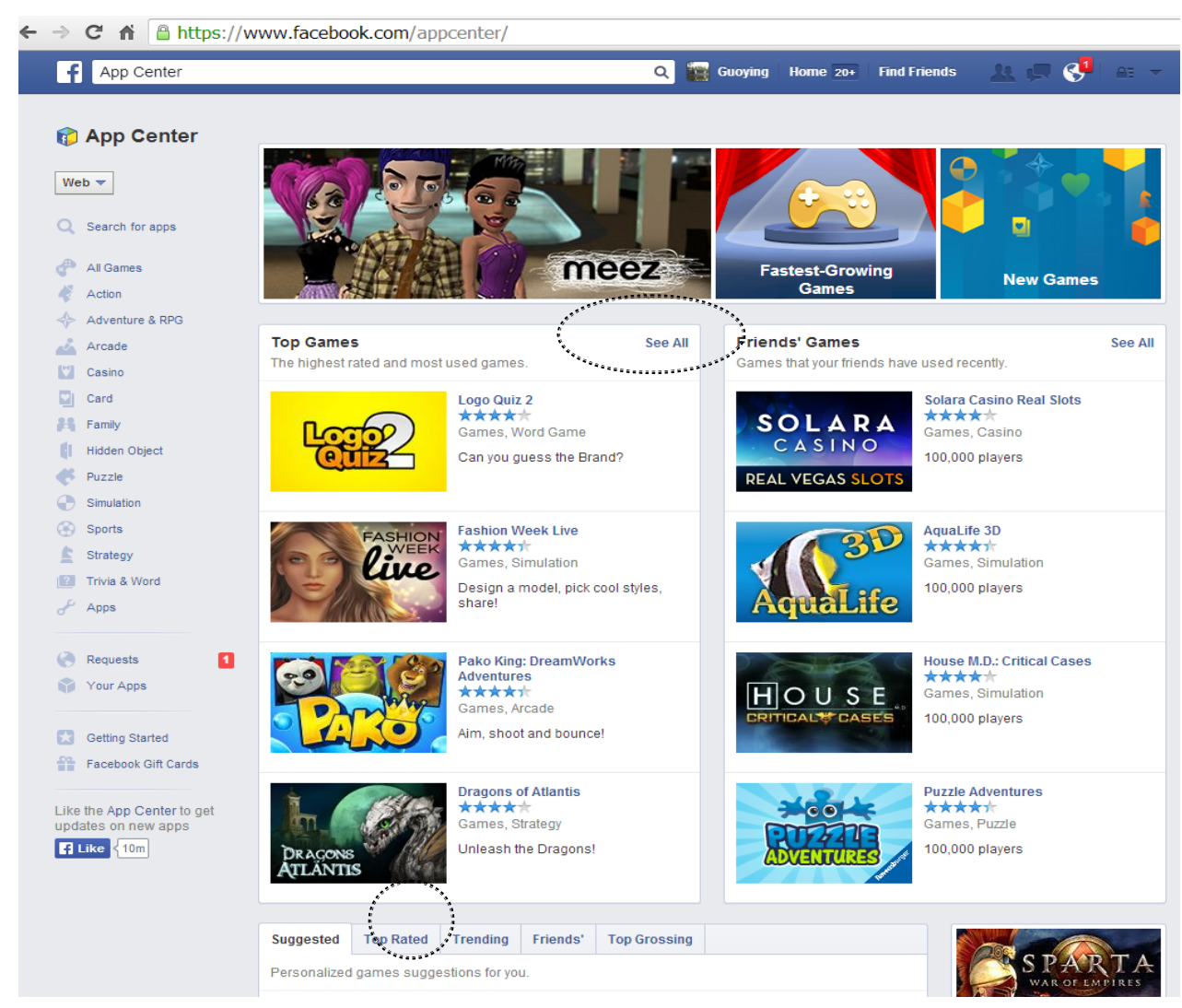




\section{Copyrights}

Copyright for this article is retained by the author(s), with first publication rights granted to the journal.

This is an open-access article distributed under the terms and conditions of the Creative Commons Attribution license (http://creativecommons.org/licenses/by/3.0/). 